\title{
Diferença entre precipitação pluvial e evapotranspiração potencial em época de colheita de uva
}

\author{
Difference between precipitation and potential evapotranspiration during grape \\ harvesting season
}

\author{
Ludmila Bardin Camparotto $\left.{ }^{1}{ }^{*}\right)$ \\ Gabriel Constantino Blain² \\ Mário José Pedro Júnior ${ }^{3}$ \\ José Luiz Hernandes ${ }^{4}$
}

\section{Resumo}

A fim de favorecer o acúmulo de açúcares nas bagas de uva, os viticultores da região de Jundiaí, Estado de São Paulo, têm realizado a denominada poda extemporânea na qual, a época de colheita ocorre dentro da estação seca regional. Nesse contexto e sob a hipótese de que as séries decendiais da diferença entre a precipitação pluvial e a evapotranspiração potencial (P-ETP) dessa localidade são formadas por dados independentes e identicamente distribuídos (iid), o objetivo do trabalho foi avaliar o ajuste de diferentes distribuições estatísticas a esse parâmetro agrometeorológico. De forma geral, os testes Run e Mann-Kendall e a análise de ondaleta indicam que as séries de P-ETP podem ser consideradas livres de correlação serial, tendências e periodicidades. Esses resultados permitiram a adoção do pressuposto iid. O teste de aderência de Kolmogorov-Smirnov/Lilliefors (KS-L) foi aplicado considerando-se as seguintes distribuições: Wakeby, Kappa, generalizada dos valores extremos, Pearson tipo III, Lognormal (com 3 parâmetros) e Logística Generalizada. Com base no KS-L e em resultados obtidos a partir de gráficos quantil-quantil, índice modificado de concordância e erro médio absoluto, conclui-se que a distribuição Lognormal pode ser utilizada para estimar a probabilidade de ocorrência associada aos valores decendiais do parâmetro P-ETP.

Palavras-chave: Probabilidade, Distribuição Lognormal, Tendência climática.

1 Dra.; Agricultura Tropical e Subtropical; Instituto Agronômico de Campinas, IAC, Brasil; Pesquisadora Visitante no Instituto Agronômico; Endereço: Av. Barão de Itapura, 1481 Guanabara, CEP:13001940 - Campinas, SP - Brasil - Caixapostal: 28; E-mail: ludmila_bardin@yahoo.com.br $\quad(*)$ Autor para correspondências

2 Dr., Eng. Agrícola, Pesquisador, Centro de Ecofisiologia e Biofísica, IAC/APTA/SAA, Av. Barão de Itapura, 1481, 13020-902, Campinas-SP, Fone: (19) 3202-1689, gabriel@iac.sp.gov.br

3 Dr., Eng. Agrônomo, Pesquisador, Centro de Ecofisiologia e Biofísica, IAC/APTA/SAA, Campinas-SP, Av. Barão de Itapura, 1481, 13020-902, Campinas-SP, Fone: (19) 3202-1689, Bolsista do CNPq, mpedro@iac.sp.gov.br

\begin{tabular}{llllll}
\hline Ambiência & Guarapuava (PR) & v.14 n.1 & p. 57-71 & Jan/Abr 2018 & ISSN $1808-0251$
\end{tabular}




\section{Abstract}

The grape growers of Jundiaí county (State of São Paulo, Brazil) are using the double pruning technique to allow harvesting during the regional dry season that favors berry sugar content. On such a background and also considering the hypothesis that the ten day series composed by the difference between precipitation and potential evapotranspiration (PEP) comprise independent and identically distributed (iid) data, the aim of this study was to evaluate the fit of several theoretical distributions to the PEP series of the location of Jundiaí, State of São Paulo, Brazil. In general, the run test, the Mann-Kendall test and the wavelet analysis indicated the presence of no serial correlation, no trend and no conclusive periodicity in these series. The Kolmogorov-Smirnov/Lilliefors (KS-L) was applied to the following distributions: Wakeby, Kappa, Generalized Extreme Value, Pearson Type III, 3- parameter LogNormal (LNG) and Generalized Logistic. From the KS-L outcomes and also based on the results obtained from the quantil-quantil plots, modified index of agreement and absolute mean error, we have concluded that the LogNormal distribution can be used to assess the probability of occurrence of the PEP values observed in the location of Jundiaí.

Key words: Probability, lognormal distribution, climate trend

\section{Introdução}

Apesar de ter como principal enfoque a produção de uvas para mesa, o viticultor do leste paulista tem voltado sua atenção para a possibilidade da produção de uva e vinho de melhor qualidade devido a demanda ocasionada pelo agroturismo. Contudo, considerando a poda normal de inverno, realizada entre julho e agosto, o período de maturação e colheita ocorre durante o auge da estação chuvosa regional (meses de dezembro e janeiro). Segundo Mota et al. (2006), essa característica climática é prejudicial ao acúmulo de açúcares e, consequentemente, à elaboração de vinho. Na tentativa de obtenção de um produto de melhor qualidade, os viticultores têm efetuado, nos meses de janeiro e fevereiro, a denominada poda extemporânea com o objetivo de realizar a colheita em época coincidente com a estação seca regional (TONIETTO e CARBONEAU, 2004; AMORIN et al., 2005; MOTA et al., 2006 e FAVERO et al., 2008).

Tonietto et al. (2006) utilizam o parâmetro do balanço hídrico determinado pela diferença entre a precipitação pluvial e a evapotranspiração potencial (P-ETP) na caracterização de meses com déficit hídrico mais elevado que, por sua vez, podem indicar condições favoráveis à maturação da uva e síntese de polifenóis. Com isso, o conhecimento da curva de probabilidade associada ao parâmetro P-ETP, em regiões produtoras de uva do leste paulista, constitui-se em informação agrometeorológica de suma importância no estabelecimento de uma viticultura regional sustentável. Sob o enfoque estatístico, a determinação da curva de probabilidade de dada variável é frequentemente realizada com base em distribuições teórico-paramétricas. Com base nos estudos de Hosking e Wallis (1997), Sen e Niedzielski (2010) e Santos et al. (2011), é possível inferir que distribuições multi-paramétricas podem ser utilizadas para representar processos naturais, tais como o parâmetro P-ETP, que são influenciados por diversos fatores. 
Por definição, a variação do parâmetro P-ETP está associada aos valores da evapotranspiração potencial ou de referência (ETP; cultura padrão) que, por sua vez, são determinados pelas variáveis associadas à demanda hídrica atmosférica. Em adição, essa variável é também fortemente influenciada pelos fatores que modulam a precipitação pluvial observada no período recortado.

Considerando as características tropicais do clima de Jundiaí, enquanto alguns anos podem apresentar um determinado decêndio (i) com valores de precipitação pluvial consideravelmente superior aos de $\operatorname{ETP}_{\mathrm{i}}\left(\mathrm{P}-\mathrm{ETP}_{\mathrm{i}}>0\right)$, em outros anos, podem ser encontrados valores nulos de precipitação no mesmo i período considerado $\left(\mathrm{P}-\mathrm{ETP}_{\mathrm{i}}=-\mathrm{ETP}_{\mathrm{i}}\right)$. Dessa forma, torna-se natural a busca de uma distribuição de probabilidade com flexibilidade suficiente para representar todos os possíveis valores que o parâmetro P-ETP pode assumir em determinada região e período.

A escolha dessa função de probabilidade requer o conhecimento prévio da existência de correlação serial ou persistência temporal na série em análise. Conforme indicado por Maia et al. (2007), o uso de funções cumulativas de probabilidade (cdf) para a descrição de uma série temporal somente é adequado para dados livres de correlação serial ou fracamente auto-correlacionados. Em séries com persistência temporal moderada a forte, o uso das referidas cdf pode acarretar perda de importantes informações (MAIA et al., 2007).

Outro aspecto importante a ser considerado é o fato de que a presença de significativas periodicidades e de tendências climáticas em uma amostra interfere em sua estrutura de probabilidade (BLAIN, 2011a). Nesse aspecto, observa-se que a utilização de distribuições teóricoparamétricas para avaliação da probabilidade de ocorrência de determinada variável baseia-se, frequentemente, no pressuposto de que os dados amostrais são independentes e identicamente distribuídos (idd) (WILLKS, 2011; EL ADLOUNI et al., 2007). Naturalmente, sob a presença de tendências significativas em uma série de dados, o pressuposto idd não pode ser adotado (CHANDLER e SCOTT, 2011).

Dessa forma, com base na hipótese de que as séries decendiais de P-ETP, obtidas a partir da estação meteorológica de Jundiaí, são formadas por dados independentes, e que a probabilidade de ocorrência associada aos diversos valores dessa variável pode ser estimada por uma função paramétrica conhecida, o objetivo do trabalho foi avaliar o ajuste de diferentes distribuições teóricas a esse parâmetro agrometeorológico. Este estudo pode servir de subsídio para tomada de decisão dos viticultores da região de Jundiaí, Estado de São Paulo, quanto à determinação da melhor época de poda, se para a safra de verão ou de inverno e, consequentemente, colheita.

\section{Material e Métodos}

Foram utilizados dados decendiais de precipitação pluvial e temperatura do ar, pertencentes ao Instituto Agronômico (IAC/SAA-SP) da localidade de Jundiaí (2311'S; 4653’W; 771m) entre 1969 a 2014. Todos os testes estatísticos foram conduzidos a 5\% de significância. No período considerado a série não apresentou falhas.

Pelos padrões normais do Estado de São Paulo, de outubro a março a precipitação excede a ETP; de abril a setembro, após equilíbrio entre essas variáveis, a ETP torna-se maior que a precipitação pluvial, resultando em períodos de deficiência hídrica em grande parte do Estado (ORTOLANI e CAMARGO, 1987).

Conforme descrito em Blain (2009) as séries mensais de precipitação pluvial da localidade de Jundiaí apresentam, durante o período de inverno, distribuições com elevados graus de 
assimetria características de regiões semi-áridas. Em contrapartida, ao longo dos meses de verão tais distribuições assumem formas próximas à Guassiana, as quais são frequentemente observadas em clima equatorial. De acordo com a classificação de Köepen, o clima local é Cfa. A ETP foi estimada por meio do método de Thonthwaite, descrito em diversos trabalhos tais como Pereira et al. (2002).

A determinação do coeficiente de autocorrelação, relativo ao primeiro deslocamento $\left(\mathrm{r}_{1}\right.$; Lag 1) foi realizada para a verificação da presença de significativa correlação serial presente nas séries temporais de P-ETP. Esse método, descrito em diversos trabalhos tais como Wilks (2011), foi aplicado em cada série decendial e em uma série que contém todos os dados decêndiais organizados em sua ordem cronológica natural (P-ETP_TOT). O ciclo sazonal (anual) dos dados de P-ETP_TOT foi removido, subtraindo-se, de cada valor, a respectiva mediana decendial (P-ETP_TOT').

A análise de ondaletas foi utilizada a fim de decompor a série de P-ETP_TOT'no domínio tempo-frequência. Dessa forma, essa análise espectral permite tanto determinar a existência de picos de variância, no domínio da frequência, quanto analisar a distribuição temporal dos mesmos. Informações detalhadas sobre a análise da ondaleta, incluindo a adoção da ondaleta mãe de Morlet e a determinação da significância estatística dos picos de energia, podem ser encontradas em Torrence e Compo (1998), Reboita et al. (2006), Blain (2009), Pezzi e Kayano (2009) e Blain e Kayano (2011) a AO. Ela foi calculada com base no algoritmo computacional descrito em http:// paos.colorado.edu/research/wavelets (acessado em 30, Novembro de 2012).

O teste de Mann-Kendall (MANN, 1945; KENDALL e STUART, 1967) foi utilizado a fim de verificar possíveis tendências, de elevação ou queda, presente nos dados. De acordo com Önöz e Bayazit (2011) esse método não paramétrico é um dos mais utilizados para detecção de tendências em séries temporais.

\section{Distribuições paramétricas}

Naturalmente, funções indefinidas para valores iguais ou inferiores a zero não foram consideradas no presente estudo. As distribuições utilizadas foram Wakeby (WAK), Kappa (KAP), generalizada dos valores extremos (GEV), Pearson tipo III (PE3; também denominada de gama 3-parâmetros), Lognormal (LNG; parametrizada conforme HOSKING E WALLIS, 1997) e Logística Generalizada (LG). As distribuições WAK e KAP possuem, respectivamente, 5 e 4 parâmetros. As demais funções de probabilidade apresentam 3 parâmetros. A LNG é descrita pela equação 1 . Descrições detalhadas sobre as demais distribuições podem ser encontradas em diversos estudos tais como Hosking e Wallis (1997).

$$
F(P-E T P)=\Phi(z)
$$

Em que $\Phi$ é a função acumulativa de densidade de probabilidade associada à distribuição normal e z é dado pela equação 2 .

$$
z=\left\{\begin{array}{l}
-\xi^{-1} \ln \left[1-\frac{\xi(\mathrm{P}-\mathrm{ETP}-\mu)}{\sigma}\right] ; \text { se } \xi \neq 0 \\
\frac{P-E T P-\mu}{\sigma} ; \text { se } \xi=0
\end{array}\right.
$$


Segundo Hosking e Wallis (1997), dependendo do valor de seus parâmetros $\xi$ (forma), $\mu$ (localização) e $\sigma$ (escala), a LNG pode representar, como casos particulares da equação 1 , as distribuições lognormal com assimetria positiva $(\xi<0)$, lognormal com assimetria negativa $(\xi>0)$ e normal $(\xi=0)$. O método dos $L$-moments $(\mathrm{lm})$ foi utilizado na estimativa dos parâmetros das distribuições, conforme recomendado por Hosking e Wallis (1997).

- Verificação do ajuste

Os testes qui-quadrado $\left(X^{2}\right)$ e Kolmogorov-Smirnov (KS) são frequentemente utilizados para verificar o ajuste de dada amostra a uma distribuição paramétrica. Entretanto, Wilks (2011) afirma que $\mathrm{o} X^{2}$ é mais apropriado para variáveis discretas, uma vez que seu cálculo exige a divisão da amostra em classes discretas de frequência de ocorrência. Em contrapartida o KS é baseado na comparação das distribuições cumulativas teóricas e empíricas (equação 3; D) sendo, portanto, mais apropriado a variáveis contínuas (WILKS, 2011).

$$
D=\left|F^{\prime}(P-E T P)-F(P-E T P)\right|
$$

Em que:

F'(P-ETP) é a frequencia acumulada empírica dos valores de P-ETP e F(P-ETP) é dado pelo distribuição teórico-paramétrica em análise.

Em sua forma original, o KS pode ser aplicado para verificar o ajuste de uma função paramétrica desde que os parâmetros dessa distribuição não tenham sido estimados a partir da mesma amostra de dados em que esse teste de aderência esteja sendo empregado (WILKS, 2011). A não observação dessa restrição pode levar à aceitação de uma $\mathrm{H}_{0}$ falsa (CRUTCHER, 1975; STEINSKOG et al., 2007; VLČEK E HUTH, 2009 e WILKS, 2011). A fim de contornar esse problema, uma modificação que frequentemente recebe o nome de Kolmogorov-Smirnov/ Lilliefors (KS-L) pode ser empregada.

Conforme descrito em diversos trabalhos, tais como Wilks (2011), valores críticos para o teste KS-L podem ser obtidos por meio de simulações estatísticas. Esse procedimento inicia-se com a geração de um elevado número (Ns) de séries sintéticas (ou simuladas; Ss), a partir de uma distribuição conhecida (WAK, KAP, GEV, LNG, GL e PE3, no presente estudo). Após essa etapa, os parâmetros de cada distribuição em estudo são estimados com base em cada série Ss. O ajuste relativo a cada série $\mathrm{Ss}$, entre os dados gerados a partir da distribuição conhecida e a função a estes ajustada, é avaliada por meio da equação 3. Conforme Wilks (2011) e Blain (2011b), adotou-se Ns=10.000. Assim, para cada distribuição foram obtidos NS valores de D (equação 3). Por definição estatística, a $\mathrm{H}_{0}$ é verdadeira para todas as $\mathrm{Ns}$ aplicações da equação 3. Assim, os 10.000 valores de Ds dão origem à distribuição de nulidade do teste KS-L a qual permite a determinação do valor crítico de rejeição de uma $\mathrm{H}_{0}$ para o nível de significância e distribuição consideradas.

Uma crítica que pode ser atribuída ao KS-L é o fato de que, embora a equação 3 quantifique a diferença entre a frequência acumulada empírica e a probabilidade teórico-cumulativa, ambos ,F'(P-ETP) e F(P-ETP) convergem ao mesmo fator comum. Em outras palavras, os dois parâmetros da equação 3 tendem a 1 conforme elevam-se os valores de P-ETP. Nesse aspecto, Coles (2001) recomenda o uso dos gráficos quantil-quantil (QQ), que, no caso do presente estudo, comparam os valores observados aos estimados de P-ETP. Para auxiliar a avaliação dos gráficos 
QQ, utilizou-se o erro médio absoluto (EMA) e o índice modificado de concordância de Willmott $\left(\mathrm{d}_{\text {mod }}\right.$; Willmott et al., 1985). Informações detalhadas sobre o EMA, que apresenta a mesma dimensão da variável sob análise, podem ser encontradas em trabalhos tais como Wilks (2011). O índice $\mathrm{d}_{\text {mod }}$ é adimensional $\exists \exists$ [0:1] em que $\mathrm{d}_{\bmod }=1$ descreve um ajuste perfeito entre os dados estimados e observados. Quando aplicados a um mesmo modelo, $\mathrm{d}_{\text {mod }}$ será sempre menor, ou no máximo igual, ao índice original de concordância de Willmott (WILLMOTT et al., 1985). O intervalo de confiança associado a cada estimativa de EMA e $\mathrm{d}_{\text {mod }}$ foi obtido por meio da técnica de bootstrap (reamostragem com reposição), conforme recomendado por Willmott et al. (1985).

\section{Resultados e Discussão}

\section{Persistência temporal, periodicidades e tendências climáticas}

Todas as séries decendiais de P-ETP podem ser consideradas livres de correlação serial (Tabela 1). Essa indicação é corroborada pelo valor não significativo de $r_{1}$ obtido para a série P-ETP_TOT $\left(r_{1}=-0,01\right)$. Dessa forma, pode-se inferir que o uso de distribuições paramétricas para a descrição probabilística das séries de P-ETP, não resultará em perdas de importantes informações devido à presença de correlações seriais (MAIA et al., 2007). A inexistência de auto-correlação significativa também é apropriada para a aplicação do MK em sua forma original (VON STORCH 1995; YUE et al., 2002).

De acordo com o MK, para a localidade de Jundiaí, apenas duas das 36 séries decendiais (Tabela 1) apresentam tendências climáticas significativas. No primeiro e segundo decêndio dos meses de Janeiro e Março, respectivamente, observa-se tendência de elevação nos valores de P-ETP. Sob o enfoque agrometeorológico, essa indicação sugere um aumento médio no suprimento hídrico às culturas. Em outras palavras, esses valores significativos do MK descrevem uma tendência para uma condição mais úmida. Em termos estatísticos, valores significativos do teste de MK não permitem a adoção da hipótese de que a amostra é formada por dados independentes e identicamente distribuídos (CHANDLER e SCOTT, 2011). Considerando o rigor científico, essa última característica diminui a consistência da parametrização dessas duas séries decendiais, uma vez que a estrutura de probabilidade delas não pode ser vista como sendo independente da escolha de uma origem temporal (COLES, 2001; EL ADLOUNI et al., 2007 e BLAIN, 2011a). 
Tabela 1 - Coeficiente de autocorrelação $\left(r_{1}\right)$ relativo ao primeiro deslocamento e teste de Mann-kendall (MK e sua respectiva significância $p$ ) aplicados a valores decendiais da diferença entre precipitação e evapotranspiração potencial da localidade de Jundiaí, Estado de São Paulo (1969-2014). Os limites críticos de significância $(5 \%)$ de $r_{1}$ são [-0.31:0.27]

\begin{tabular}{ccccc}
\hline Mês & Decêndio & $\mathbf{r}_{1}$ & $\mathbf{M K}$ & $\mathbf{p}$ \\
\hline Janeiro & 1 & 0,13 & $2,45^{*}$ & 0,01 \\
Janeiro & 2 & $-0,11$ & 0,36 & 0,72 \\
Janeiro & 3 & $-0,23$ & 1,11 & 0,27 \\
Fevereiro & 4 & 0,19 & 0,02 & 0,98 \\
Fevereiro & 5 & 0,09 & 1,01 & 0,31 \\
Fevereiro & 6 & $-0,05$ & 0,18 & 0,86 \\
Março & 7 & $-0,03$ & $-0,32$ & 0,75 \\
Março & 8 & $-0,08$ & $2,25^{*}$ & 0,02 \\
Março & 9 & $-0,16$ & 0,00 & $>0,99$ \\
Abril & 10 & 0,03 & $-1,50$ & 0,13 \\
Abril & 11 & 0,25 & $-0,59$ & 0,56 \\
Abril & 12 & 0,04 & 0,24 & 0,81 \\
Maio & 13 & $-0,04$ & 0,34 & 0,73 \\
Maio & 14 & 0,23 & 0,20 & 0,84 \\
Maio & 15 & 0,03 & 0,20 & 0,84 \\
Junho & 16 & 0,02 & $-0,04$ & 0,97 \\
Junho & 17 & $-0,02$ & $-0,79$ & 0,43 \\
Junho & 18 & $-0,21$ & 0,16 & 0,87 \\
Julho & 19 & 0,05 & $-1,31$ & 0,19 \\
Julho & 20 & $-0,06$ & 1,01 & 0,31 \\
Julho & 21 & 0,04 & $-0,22$ & 0,82 \\
Agosto & 22 & $-0,06$ & $-0,20$ & 0,84 \\
Agosto & 23 & $-0,16$ & $-1,19$ & 0,23 \\
Agosto & 24 & $-0,11$ & $-0,16$ & 0,87 \\
Setembro & 25 & 0,01 & $-1,05$ & 0,29 \\
Setembro & 26 & 0,03 & $-0,75$ & 0,45 \\
Setembro & 27 & $-0,11$ & $-0,81$ & 0,42 \\
Outubro & 28 & 0,15 & $-1,82$ & 0,07 \\
Outubro & 29 & 0,05 & $-0,91$ & 0,36 \\
Outubro & 30 & $-0,10$ & 1,78 & 0,08 \\
Novembro & 31 & $-0,14$ & $-1,38$ & 0,17 \\
Novembro & 32 & 0,01 & 0,06 & 0,95 \\
Novembro & 33 & 0,04 & 0,36 & 0,72 \\
Dezembro & 34 & 0,00 & 0,36 & 0,72 \\
Dezembro & 35 & $-0,02$ & 0,63 & 0,53 \\
Dezembro & 36 & $-0,24$ & 0,00 & $>0,99$ \\
\hline as\% & & & &
\end{tabular}

* significativo à $5 \%$ 
A análise global da ondaleta conduzida apenas no domínio da frequência (Figura 1b; GWP) descreve dois picos significativos de variância situados entre as frequências 0,33 e 0,17 ano ${ }^{-1}$. Em uma primeira análise, essa observação pode levar a indicação de significativas periodicidades no sinal temporal P-ETP. Entretanto, ao estender essa análise espectral ao domínio tempo-frequência (Figura 1a; WPS), observa-se que essa variabilidade descrita pela GWP é o resultado de um pico significativo de energia situado, exclusivamente, entre 1975 e 1990. Após essa última data, não há manifestações de significativas variâncias (ou energia) nestas referidas frequências. Dessa forma, a $\mathrm{AO}$ não permite a indicação conclusiva da presença de periodicidades no sinal temporal P-ETP. A inexistência de periodicidades no sinal temporal P-ETP também foi observada por Blain (2011a) para a localidade de Campinas (1890-2010).

Figura 1 - a) Espectro de energia local da ondaleta (WPS) para a diferença entre a precipitação pluvial e evapotranspiração potencial de Jundiaí-SP. Contornos tracejados correspondem a valores de variância normalizados $\left(1 / 1979 \mathrm{~mm}^{2}\right)$ variando de 1 a 10, conforme representado na barra horizontal. Contornos sombreados englobam áreas com variâncias significativas ao nível de $95 \%$ de confiança. A curva em forma de $U$ representa o cone de influência, sob a qual $o$ efeito de borda é importante; b) Espectro global de ondaleta (GWP).
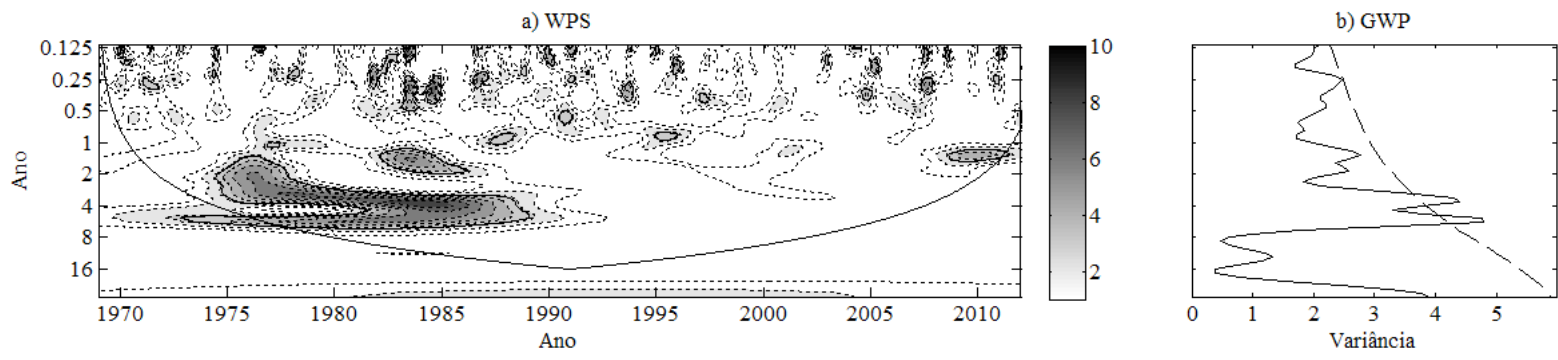

Assim, a partir da exceção das duas séries decendiais em que foi detectada presença de tendências significativas, pode-se inferir que os resultados obtidos neste estudo suportam a adoção da hipótese de que o uso das distribuições paramétricas, propostas no presente estudo, não resultará em perdas de relevantes informações do processo sobre investigação, uma vez comprovado o ajuste aos dados empíricos.

\section{Parametrização}

Segundo Wilks (2011), Haddad e Rahman (2011) e Shin et al. (2012), testes como o KS-L quantificam o ajuste entre a distribuição empírica acumulativa e a função paramétrica cumulativa. Dessa forma, esse teste fornece evidências empíricas para aceitação ou rejeição da hipótese de que uma dada distribuição teórica pode ser utilizada para avaliar a probabilidade de ocorrência do parâmetro em estudo. Dentre todas as distribuições avaliadas, a LNG foi a única em que essa hipótese $\mathrm{H}_{0}$, associada ao KS-L, foi aceita para todas as séries decendiais. Comparando-se os valores da equação 4 e os respectivos valores críticos de rejeição da $\mathrm{H}_{0}$ (Tabela 2), nota-se que a 
LNG pode, a 5\% de significância, ser utilizada para a descrição das probabilidades de ocorrência associadas aos dados de P-ETP.

Tabela 2 - Teste Kolmogorov-Smirnov/Lilliefors (KS-L) e respectivos valores críticos, calculados à $5 \%$ de significância (KScrit), valores calculados do índice de Willmott (concordância) modificado (dmod) e do erro absoluto médio (EMA) e os respectivos intervalos de confiança superiores (ICsup) e inferiores (ICinf) estimados $95 \%$ de confiabilidade

\begin{tabular}{|c|c|c|c|c|c|c|c|c|c|}
\hline \multirow{2}{*}{ Mês } & \multirow{2}{*}{ Dec } & \multirow{2}{*}{ KS-L } & \multirow{2}{*}{ KScrit } & \multicolumn{3}{|c|}{ dmod - Admensional } & \multicolumn{3}{|c|}{ EMA - (mm) } \\
\hline & & & & ICinf & dmod & ICsup & ICinf & EMA & ICsup \\
\hline Janeiro & 1 & 0,077 & 0,101 & 0,91 & 0,93 & 0,95 & 4,5 & 6,0 & 7,8 \\
\hline Janeiro & 2 & 0,080 & 0,104 & 0,91 & 0,93 & 0,95 & 4,5 & 6,5 & 8,9 \\
\hline Janeiro & 3 & 0,062 & 0,099 & 0,90 & 0,92 & 0,94 & 4,6 & 7,1 & 10,3 \\
\hline Fevereiro & 4 & 0,086 & 0,102 & 0,87 & 0,89 & 0,92 & 7,1 & 10,1 & 13,6 \\
\hline Fevereiro & 5 & 0,092 & 0,099 & 0,89 & 0,91 & 0,93 & 4,6 & 6,0 & 7,6 \\
\hline Fevereiro & 6 & 0,082 & 0,099 & 0,91 & 0,93 & 0,94 & 3,3 & 4,2 & 5,3 \\
\hline Março & 7 & 0,068 & 0,100 & 0,91 & 0,93 & 0,94 & 3,6 & 4,4 & 5,2 \\
\hline Março & 8 & 0,062 & 0,103 & 0,92 & 0,94 & 0,95 & 2,6 & 4,1 & 6,2 \\
\hline Março & 9 & 0,085 & 0,104 & 0,90 & 0,92 & 0,94 & 3,9 & 5,3 & 6,9 \\
\hline Abril & 10 & 0,069 & 0,102 & 0,92 & 0,94 & 0,95 & 1,8 & 2,6 & 3,6 \\
\hline Abril & 11 & 0,042 & 0,105 & 0,92 & 0,94 & 0,96 & 1,2 & 2,2 & 3,6 \\
\hline Abril & 12 & 0,046 & 0,111 & 0,90 & 0,93 & 0,97 & 0,8 & 2,3 & 4,2 \\
\hline Maio & 13 & 0,066 & 0,105 & 0,90 & 0,93 & 0,94 & 1,8 & 2,7 & 3,7 \\
\hline Maio & 14 & 0,117 & 0,104 & 0,91 & 0,93 & 0,95 & 1,8 & 2,4 & 3,0 \\
\hline Maio & 15 & 0,079 & 0,113 & 0,89 & 0,91 & 0,93 & 3,2 & 5,1 & 7,4 \\
\hline Junho & 16 & 0,084 & 0,119 & 0,89 & 0,91 & 0,94 & 2,3 & 4,4 & 7,1 \\
\hline Junho & 17 & 0,079 & 0,113 & 0,90 & 0,93 & 0,96 & 1,3 & 2,7 & 4,6 \\
\hline Junho & 18 & 0,090 & 0,110 & 0,89 & 0,92 & 0,94 & 2,0 & 2,9 & 4,0 \\
\hline Julho & 19 & 0,088 & 0,123 & 0,83 & 0,88 & 0,94 & 1,1 & 3,4 & 7,2 \\
\hline Julho & 20 & 0,108 & 0,111 & 0,85 & 0,88 & 0,91 & 2,9 & 4,7 & 6,7 \\
\hline Julho & 21 & 0,064 & 0,122 & 0,87 & 0,91 & 0,94 & 1,9 & 3,7 & 6,1 \\
\hline Agosto & 22 & 0,085 & 0,116 & 0,88 & 0,91 & 0,93 & 1,3 & 2,1 & 3,1 \\
\hline Agosto & 23 & 0,107 & 0,117 & 0,87 & 0,90 & 0,92 & 1,2 & 2,4 & 4,4 \\
\hline Agosto & 24 & 0,068 & 0,120 & 0,87 & 0,91 & 0,94 & 1,6 & 3,4 & 6,0 \\
\hline Setembro & 25 & 0,097 & 0,106 & 0,88 & 0,91 & 0,93 & 3,5 & 4,9 & 6,5 \\
\hline Setembro & 26 & 0,058 & 0,108 & 0,90 & 0,93 & 0,95 & 1,9 & 2,9 & 4,1 \\
\hline Setembro & 27 & 0,074 & 0,107 & 0,91 & 0,93 & 0,96 & 1,7 & 3,3 & 5,5 \\
\hline Outubro & 28 & 0,063 & 0,101 & 0,93 & 0,95 & 0,96 & 2,2 & 2,8 & 3,4 \\
\hline Outubro & 29 & 0,091 & 0,102 & 0,89 & 0,92 & 0,94 & 3,7 & 5,1 & 6,6 \\
\hline Outubro & 30 & 0,043 & 0,103 & 0,92 & 0,94 & 0,95 & 2,2 & 3,4 & 4,7 \\
\hline Novembro & 31 & 0,067 & 0,104 & 0,90 & 0,92 & 0,94 & 2,3 & 3,5 & 4,9 \\
\hline Novembro & 32 & 0,057 & 0,099 & 0,93 & 0,95 & 0,96 & 2,7 & 3,9 & 5,4 \\
\hline Novembro & 33 & 0,065 & 0,100 & 0,93 & 0,94 & 0,95 & 3,2 & 4,2 & 5,4 \\
\hline
\end{tabular}




\begin{tabular}{llllllllll} 
Dezembro & 34 & 0,048 & 0,100 & 0,94 & 0,95 & 0,96 & 2,5 & 3,5 & 4,8 \\
Dezembro & 35 & 0,092 & 0,099 & 0,90 & 0,92 & 0,94 & 4,0 & 5,1 & 6,2 \\
Dezembro & 36 & 0,062 & 0,098 & 0,92 & 0,93 & 0,95 & 3,8 & 4,6 & 5,5 \\
\hline
\end{tabular}

Essa última inferência, baseada no KS-L, é coerente com os resultados obtidos por meio do EMA e do $d_{\text {mod }}$ (Tabela 2). Considerando o valor inferior do IC associado ao $\mathrm{d}_{\text {mod }}$, nota-se que os valores desse índice permanecem superiores à 0,83 ; indicando boa concordância entre os dados observados e estimados de P-ETP por meio da LNG. A mesma inferência pode ser atribuída ao EMA. Para essa medida escalar de acurácia, mesmo considerando-se os valores superiores do IC, nota-se que em apenas 2 decêncios o EMA ultrapassa $10 \mathrm{~mm}$ (Tabela 2). Em adição, nota-se que no período da colheita de inverno, (maio a junho) o maior valor de EMA é 3,4 mm (associado à um IC superior de 7,2 mm; Tabela 2).

Dessa forma, torna-se possível aceitar que a função associada à LNG pode ser utilizada, com satisfatória confiabilidade, para a determinação das probabilidades de ocorrência associadas aos dados decendiais de P-ETP. Nesse aspecto, ressalta-se que os decêndios em que o pressuposto idd não pôde ser aceito (primeiro e segundo decêndio dos meses de janeiro e abril, respectivamente), estão temporalmente distantes das épocas de colheita, para a safra de inverno, da uva na região de Jundiaí, Estado de São Paulo. Nota-se também que as distribuições decendiais de P-ETP apresentam assimetria positiva, uma vez que o parâmetro $\xi$ apresenta valores negativos em todos os períodos (Tabela 3; HOSKING e WALLIS, 1997). Por fim, ressalta-se que os valores mais baixos do parâmetro $\mu$, associado a medidas de posição central, são observados no mês de agosto. Essa característica é coerente com os resultados obtidos por Blain (2009) que apontam que esse referido período constituí-se no auge da estação seca regional. 
Tabela 3 - Parâmetros $\xi$ (forma), $\mu$ (localização) e $\sigma$ (escala) da distribuição Lognormal, parametrizada conforme Hosking \& Wallis (1997), obtidos a partir de dados decendiais da diferença entre a precipitação pluvial e evapotranspiração potencial de Jundiaí-SP

\begin{tabular}{ccccc}
\hline Mês & Decêndio & $\boldsymbol{\mu}$ & $\boldsymbol{\sigma}$ & $\boldsymbol{\xi}$ \\
\hline Janeiro & 1 & 38,64 & 51,17 & $-0,43$ \\
Janeiro & 2 & 25,63 & 54,67 & $-0,53$ \\
Janeiro & 3 & 32,11 & 57,62 & $-0,32$ \\
Fevereiro & 4 & 10,31 & 56,20 & $-0,43$ \\
Fevereiro & 5 & 26,64 & 43,80 & $-0,13$ \\
Fevereiro & 6 & 14,36 & 35,89 & $-0,22$ \\
Março & 7 & 10,03 & 38,30 & $-0,25$ \\
Março & 8 & 10,84 & 39,69 & $-0,50$ \\
Março & 9 & $-1,48$ & 37,02 & $-0,57$ \\
Abril & 10 & $-4,68$ & 25,36 & $-0,45$ \\
Abril & 11 & $-7,18$ & 21,81 & $-0,61$ \\
Abril & 12 & $-13,00$ & 16,67 & $-0,87$ \\
Maio & 13 & $-4,06$ & 19,48 & $-0,62$ \\
Maio & 14 & $-4,21$ & 20,20 & $-0,52$ \\
Maio & 15 & 0,39 & 26,33 & $-0,92$ \\
Junho & 16 & $-6,91$ & 20,26 & $-1,06$ \\
Junho & 17 & $-6,94$ & 17,59 & $-0,91$ \\
Junho & 18 & $-4,47$ & 17,06 & $-0,81$ \\
Julho & 19 & $-10,70$ & 10,97 & $-1,09$ \\
Julho & 20 & $-5,61$ & 18,32 & $-0,87$ \\
Julho & 21 & $-11,36$ & 14,94 & $-1,12$ \\
Agosto & 22 & $-13,78$ & 9,21 & $-1,01$ \\
Agosto & 23 & $-16,04$ & 9,75 & $-1,03$ \\
Agosto & 24 & $-17,64$ & 14,48 & $-1,08$ \\
Setembro & 25 & $-3,84$ & 28,32 & $-0,66$ \\
Setembro & 26 & $-11,93$ & 21,27 & $-0,72$ \\
Setembro & 27 & $-4,95$ & 27,20 & $-0,66$ \\
Outubro & 28 & 4,57 & 33,30 & $-0,34$ \\
Outubro & 29 & 7,04 & 34,33 & $-0,50$ \\
Outubro & 30 & $-2,75$ & 32,04 & $-0,49$ \\
Novembro & 31 & $-2,92$ & 26,05 & $-0,53$ \\
Novembro & 32 & 24,59 & 46,20 & $-0,11$ \\
Novembro & 33 & 20,69 & 44,87 & $-0,27$ \\
Dezembro & 34 & 22,61 & 46,36 & $-0,26$ \\
Dezembro & 35 & 34,67 & 41,88 & $-0,05$ \\
Dezembro & 36 & 33,19 & 44,41 & $-0,07$ \\
\hline
\end{tabular}


É apresentado, na Figura 2, um exemplo de aplicação prática dos resultados obtidos no presente estudo, em que a probabilidade acumulada de ocorrência associada à $\mathrm{P}-\mathrm{ETP}=0$ é estimada para cada decêndio. O presente exemplo considerou as safras de verão e inverno, para o cultivo de uvas rústicas, tais como Bordo e Isabel, e viníferas tais como Syrah e Cabernet Sauvignon. As datas de poda, duração do ciclo e épocas de maturação relativas a cada um desses casos são apresentadas na Figura 2. As mesmas coincidem com o calendário agrícola regional. Por fim, ressalta-se que a Figura 2 pode ser elaborada para qualquer valor de interesse de P-ETP.

\section{Figura 2 - Probabilidade acumulada de ocorrência associada a dados decendiais da diferença entre a precipitação pluvial e evapotranspiração potencial de Jundiaí-SP.}

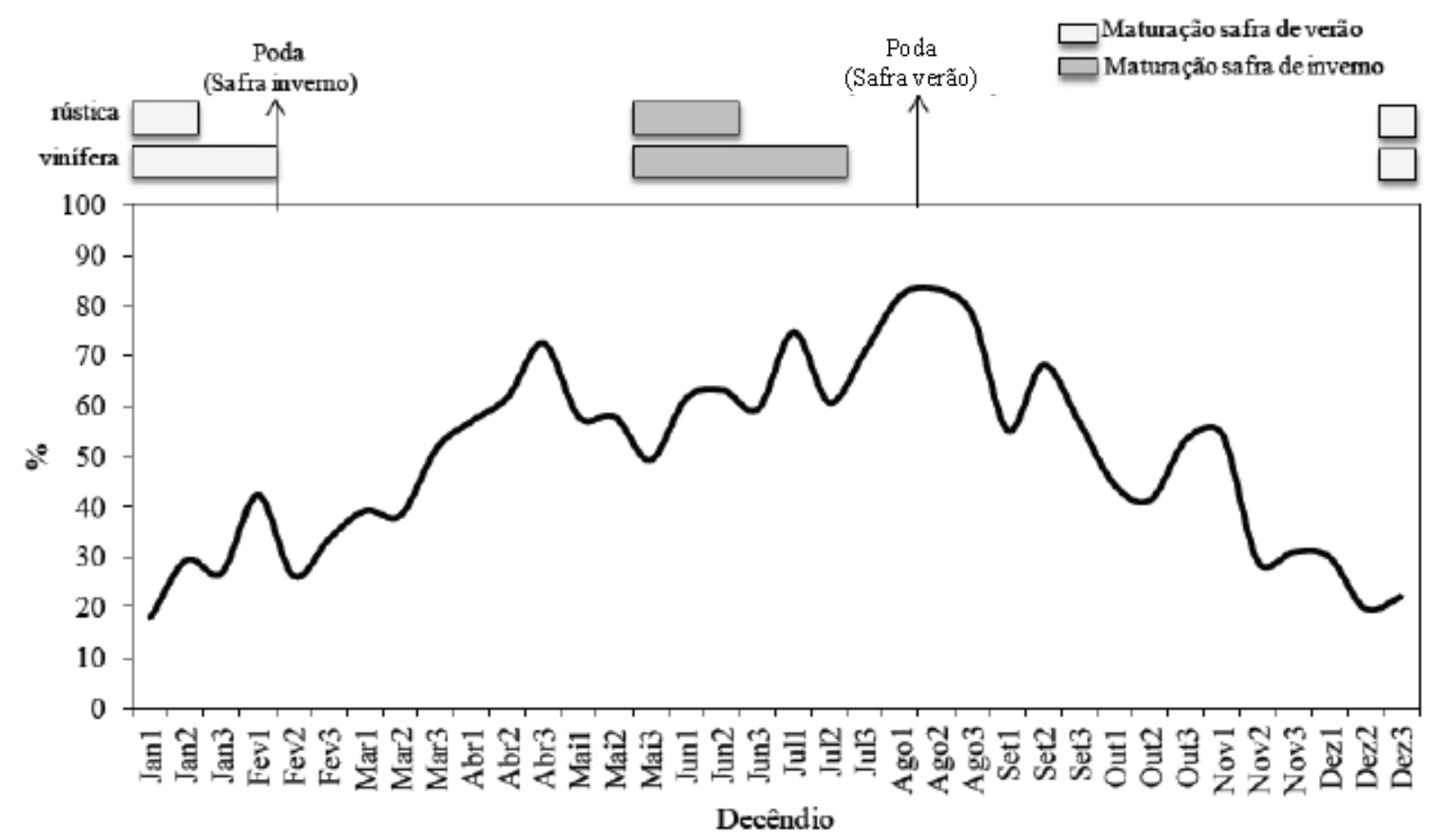

Para a safra de verão, o período de maturação de uvas rústicas (Figura 2) ocorre em decêndios nos quais a probabilidade acumulada de ocorrência $\operatorname{Pr}[\mathrm{x} \leq \mathrm{P}-\mathrm{ETP}=0]$ varia entre 15 e $30 \%$. Para o caso de uvas viníferas (ciclo aproximado de 180 dias), o período de maturação ocorre entre meados de dezembro e fevereiro. À semelhança do observado para uvas rústicas na referida safra, o período de maturação e colheita também coincide com decêndios com baixa probabilidade de o valor de precipitação pluvial ser igual ou inferior ao da evapotranspiração potencial. Naturalmente, essa condição climática úmida, observada tanto para uvas rústicas quanto viníferas, pode ser considerada desfavorável ao processo de acúmulo de açúcar nas bagas e, consequentemente, a qualidade final do vinho.

Para a safra de inverno (poda extemporânea), verifica-se que o período aproximado da maturação para uvas rústicas e viníferas ocorre em decêndios com $\operatorname{Pr}[\mathrm{x} \leq \mathrm{P}-\mathrm{ETP}=0]$ entre 50 e 85\% (Figura 2). Naturalmente, esse resultado indica uma condição climatológica mais seca e, portanto, potencialmente mais propícia ao processo de acúmulo de açúcares ao longo do período de maturação. Dessa forma, a realização da poda extemporânea, na localidade de Jundiaí, torna-se 
recomendável para a colheita de uvas para vinho, uma vez que assim, será agregado maior qualidade e valor ao produto final. Já em relação à uva rústica, não houve diferença significativa de qualidade considerando as uvas obtidas nas duas safras. Em adição, a análise da Figura 2 evidencia que a adoção de épocas de podas mais tardias e/ou demais formas de manejo agrícola, que resultem na ocorrência da maturação e colheita em decêndios mais próximos ao auge da estação seca regional poderá favorecer a qualidade final do vinho produzido na região de Jundiaí.

\section{Conclusão}

As séries decendiais da diferença entre a precipitação pluvial e a evapotranspiração potencial da localidade de Jundiaí, no Estado de São Paulo, não apresentam significativas persistências temporais e periodicidades em seus dados. Apenas 2 dos 36 decêndios apresentaram tendências climáticas significativas de elevação.

A distribuição lognormal, conforme descrita neste estudo, pode ser utilizada para estimar a probabilidade de ocorrência associada aos valores decendiais do parâmetro agrometeorológico em estudo.

A poda extemporânea é indicada na localidade de Jundiaí para a colheita de uvas para vinho. Já para as uvas rústicas, não houve diferença significativa de qualidade considerando as duas podas.

\section{Referências}

AMORIN, D. A.; FAVERO, A. C.; REGINA, M. A. Produção extemporânea da videira cultivar Syrah, nas condições do sul de Minas Gerais. Revista Brasileira de Fruticultura, Jaboticabal, v.27, n.2, p. 327-331, 2005.

BLAIN, G. C. Considerações estatísticas relativas à oito séries de precipitação pluvial da Secretaria de Agricultura e Abastecimento do Estado de São Paulo. Revista Brasileira de Meteorologia, São José dos Campos, v.24, n.1, p.12-23, 2009

BLAIN, G. C. Aplicação do conceito do índice padronizado de precipitação à série decendial da diferença entre precipitação pluvial e evapotranspiração potencial. Bragantia, Campinas, v.70, n.1, p. 234-245, 2011a.

BLAIN, G. C. Cento e vinte anos de totais extremos de precipitação pluvial máxima diária em Campinas, Estado de São Paulo: análises estatísticas. Bragantia, Campinas, v.70, n.3, p.722-728, 2011b.

BLAIN, G. C.; KAYANO, M. T. 118 anos de dados mensais do Índice Padronizado de Precipitação: série meteorológica de Campinas, Estado de São Paulo, Revista Brasileira de Meteorologia, São José dos Campos, v.26, n.1, p. 137-149, 2011.

CHANDLER, R. E.; SCOTT, M. E. Statistical methods for trend detection and analysis in the environmental analysis. $1^{\text {st }} \mathrm{ed}$. Chichester: John Wiley \& Sons, 2011.

COLES, S. An introduction to statistical modeling of extreme value, London: Springer, 
CRUTCHER, H. L. A note on the possible misuse of the Kolmogorov-Smirnov test. Journal Applied Meteorology, Boston, v.14, p.1600-1603, 1975.

EL ADLOUNI, S.; OUARDA, T. B. M. J.; ZHANG, X.; ROY, R.; BOBÉE, B. Generalized maximum likelihood estimators for the nonstationary generalized extreme value model. Water Resources Research, Washington, v.43, n.3, p.1-13, 2007.

FÁVERO, A. C.; AMORIM, D. A.; MOTA, R. V.; SOARES, A. M.; REGINA, M. A. Viabilidade de produção da videira 'Syrah', em cic lo de outono inverno, na região de Minas Gerais. Revista Brasileira de Fruticultura, Jaboticabal,v. 30, n. 3, p. 685-690, 2008.

HADDAD, K., RAHMAN, A. Selection of the best fit flood frequency distributionand parameter estimation procedure: a case study for Tasmania in Australia. Stochastic Environmental Research and Risk Assessment, Londres, v.25, n.3, p.415-428, 2011.

HOSKING, J. R. M.; WALLIS, J. R. Regional Frequency Analysis, Cambridge: Cambridge University Press, 1997. 224p.

KENDALL, M. A.; STUART, A. The advanced theory of statistics. $2^{\text {nd }}$ ed. Londres: Charles Griffin, 1967.

MAIA, A. H. N.; MEINKE, H.; LENNOX, S.; Stone, R. C. Inferential, non-parametric statistics to assess quality of probabilistic forecast systems. Monthly Weather Review, Boston, v.135, n.2, p.351-362, 2007.

MANN, H. B. Non-parametric tests against trend. Econometrica, Nova York, v.13, n.3, p.245-259, 1945.

MOTA, R. V.; REGINA, M.A.; AMORIN, D. A.; FÁVERO, A. C. Fatores que afetam a maturação e a qualidade da uva para vinificação. Informe Agropecuário, Belo Horizonte, v.27, n.234, p.56-64, 2006.

ÖNÖZ, B.; BAYAZIT, M. Block bootstrap for Mann-Kendall trend test of serially dependent data. Hydrogical Processes, West Lafayette, v.26, n.15, p.1-19, 2011.

ORTOLANI, A. A.; CAMARGO, M. B. P.Influência dos fatores climáticos na produção: Ecofisiologia da Produção Agrícola. Piracicaba: Potafos, 1987. 249 p.

PEREIRA, A. R.; ANGELOCCI, L. R.; SENTELHAS, P. C. Agrometeorologia: fundamentos e aplicações práticas. Guaíba: Agropecuária, 2002.

PEZZI, L. P.; KAYANO, M. T. An analysis of the seasonal precipitation forecasts in South America using wavelets. International Journal of Climatology, West Sussex, v.29, 
n.11, p.1560-1573, 2009.

REBOITA, M. S.; KRUSCHE, N.; PICCOLI, H. C. Climate variability in Rio Grande, RS, Brazil: A quantitative analysis of contributions due to atmospheric systems. Revista Brasileira de Meteorologia, Jaboticabal, v.21, p. 256-270, 2006.

SANTOS,J. F.; PORTELA, M. M.; PULIDO-CALVO, I. Regional Frequency Analysis of Droughts in Portugal. Water Resources Manage, v.25, p.3537-3558, 2011.

SEN, A. K.; NIEDZIELSKI, T. Statistical characteristics of riverflow variability in the Odra River Basin, Southwestern Poland. Polish Journal of Environmental Studies, v.19, p.387-397, 2010.

SHIN, H.; JUNG, Y.; JEONG, C. Assessment of modified Anderson-Darling test statistics for the generalized extreme value and generalized logistic distributions. Stochastic Environmental Research Risk Assessment, Londres, v.26, n.1, p.105-114, 2012.

STEINSKOG, D.J.; TJØSTHEIM, D. B.; KVAMSTØ, N. G. A cautionary note on the use of the Kolmogorov-Smirnov test for normality. Monthly Weather Review, Boston, v.135, n.3, p.1151-1157, 2007.

TONIETTO, J.; CARBONNEAU, A. A multicriteria climatic classification system for grape-growing regions worlwide. Agricultural and Forest Meteorology, Amsterdã, v. 124, p. 81-97, 2004.

TONIETTO,J.; VIANELLO, R. L.; REGINA,M.A. Caracterização macroclimática e potencial enológico de diferentes regiões com vocação vitícola de Minas Gerais. Informe Agropecuário, Belo Horizonte, v.27, n.234, p. 32-55, 2006.

TORRENCE, C.; COMPO, G. P. Practical Guide to Wavelet Analysis, Bulletins of American Meteorological Society, Boston, v.79, n.1, p.61-78, 1998.

VLČEK, O.; HUTH R. Is daily precipitation Gamma-distributed? Adverse effects of an incorrect application of the Kolmogorov-Smirnov test. Atmospheric Research, Aubière, v.93, n.4, p.759-766, 2009.

VON STORCH, H.; NAVARRA, A. Analysis of Climate Variability: Applications of Statistical Techniques. Berlin: Springer, 1995.

WILKS, D. S. Statistical methods in the atmospheric sciences. $3^{\text {rd }}$ ed. San Diego: Academic Press, 2011.

WILLMOT, C. J.; ACKLESON, S. G.; DAVIS, R. E.; FEDDEMA, J.J.; Klink, K. M.; Legates, D. R.; O'Donnell, J.; Rowe, C. M. Statistics for the evaluation and comparison of models. Journal of Geophysical Research, Newark, v.90, n.C5, p.8995-9005, 1985. 